\title{
Mechanical Regulation of Skeletal Development
}

\author{
Rebecca Rolfe $^{1}$, Karen Roddy ${ }^{1 \#}$ and Paula Murphy ${ }^{1 *}$
}

1. Department of Zoology, School of Natural Sciences, and Trinity Centre for Bioengineering, Trinity College Dublin, Ireland.

*Address for correspondence: Dr. Paula Murphy, School of Natural Sciences, Trinity College

Dublin, Ireland. Phone: +353-1-896-3780; Fax: +353-677-8094; Email:paula.murphy@tcd.ie

\# Present address: Cardiff School of Biosciences, Life Sciences Building, Museum Avenue, Cardiff, CF10 3AX

Edited and resubmitted to Current Osteoporosis Reports, February 6th 2013

Key words: skeletal development; mechanical stimulation; bone; ossification; joint; chondrogenesis; articular cartilage; regenerative therapies; mechanotransduction 


\begin{abstract}
Development of the various components of a normal skeleton requires highly regulated signalling systems that co-ordinate spatial and temporal patterns of cell division, cell differentiation and morphogenesis. Much work in recent decades has revealed cascades of molecular signalling, acting through key transcription factors to regulate, for example, organised chondrogenic and osteogenic differentiation. It is now clear that mechanical stimuli are also required for aspects of skeletogenesis but very little is known about how the mechanical signals are integrated with classic biochemical signalling. Spatially organised differentiation is vital to the production of functionally appropriate tissues contributing to precise, region specific morphologies, for example transient chondrogenesis of long bone skeletal rudiments, which prefigures osteogenic replacement of the cartilage template, compared to the production of permanent cartilage at the sites of articulation. Currently a lack of understanding of how these tissues are differentially regulated hampers efforts to specifically regenerate stable bone and cartilage. Here we review current research revealing the influence of mechanical stimuli on specific aspects of skeletal development and refer to other developing systems to set the scene for current and future work to uncover the molecular mechanisms involved. We integrate this with a brief overview of the effects of mechanical stimulation on stem cells in culture bringing together developmental and tissue engineering aspects of mechanoregulation of cell behaviour. A better understand of the molecular mechanisms that link mechanical stimuli to transcriptional control guiding cell differentiation will lead to new ideas about how to effectively prime stem cells for tissue engineering and regenerative therapies.
\end{abstract}


To better understand the basis of degenerative skeletal diseases such as osteoporosis and osteoarthritis we not only need to uncover mechanisms of bone and cartilage maintenance, but also the molecules and cues that drive skeletal formation in the embryo. A deep understanding of developmental processes is also required to harness the potential of stem cells to regenerate bone and cartilage; we need to know which stimuli are required to prime site specific, functionally appropriate, differentiation pathways. Such therapies hold great promise however it is clear that we are still some distance away from knowing how to guide the formation of functionally appropriate bone and cartilage to replace injured and degenerating tissue.

The central role of mechanical stimuli on bone maintenance and adaptation has been a fundamental part of bone biology since Wolff's Law was formulated in the $19^{\text {th }}$ century, and was incorporated by Harold Frost into his Mechanostat theory where local mechanical effects are integrated with and adjusted by the biochemical system[1, 2]. Although the effect of mechanical stimulation on the formation of the skeletal system in the embryo has been less widely considered in the past (reviewed in[3]), evidence has existed for some time that mechanical forces generated by embryonic muscle contractions are required for normal skeletogenesis. Such evidence comes from two sources; congenital muscle disorders where foetal muscle contractions are reduced in utero and from the results of experimental immobilisation of model animals. Recent work is beginning to show how mechanical stimuli impact specific developmental processes. As suggested by Frost in the Mechanostat theory, these mechanical influences must be integrated with molecular processes at a cellular level. Although more work is required to reveal the points of integration between biophysical and biochemical signals guiding skeletal development, discoveries revealing the impact of biophysical stimuli on other developing systems are providing valuable clues. The stage is now set for synergistic advances on the question of mechanoregulation of skeletal development. Here we review current research revealing the influence of mechanical stimuli on skeletal development, specifically outlining the developmental processes that are impacted, the influence on progenitor cells in culture as well as principles emerging from other developing systems. This work will prime future research to address key challenges in maintaining healthy skeletal tissue and improving regenerative capacities.

\section{Mechanical influences on developmental processes}

Our understanding of how tissue differentiation is controlled in the developing embryo and how shape and structure is established has progressed enormously in recent years. A 
picture has emerged of proliferating progenitor cells responding to layers of positional information that guide cellular differentiation and tissue morphogenesis with an emphasis on the role played by locally produced molecular signals and the response of cells through activation of specific sets of transcription factors. In more recent years it has also become apparent that we must expand these ideas to incorporate evidence that the molecular pathways are tightly integrated with physical cues and mechanical signals experienced by the developing cell[ 4,5$]$. For example, elegant work on mesenchymal condensations during tooth development has shown that cell compression is a necessary step for the modulation of gene expression and cell differentiation in response to classic signaling from epithelium to mesenchyme [6•]. Several recent studies on Drosophila development have shown the importance of mechanical signals in cell polarization, in particular impacting the Planar Cell Polarity (PCP) pathway, which shares components of the Wnt signaling pathway[7, 8]

\section{Bone and joint development and the influence of biophysical stimuli from muscle contractions}

Limb skeletogenesis begins with the condensation of prechondrogenic mesenchymal cells at the core of the limb bud in a y shaped pattern, prefiguring the future rudiments; e.g. humerus, radius and ulna in the case of the forelimb, with more distal elements added progressively[9]. Chondrogenic differentiation begins to define the individual rudiments, and the future elbow/knee joint region becomes visibly distinct, composed of flatter more dense cells; the interzone[10-12]. The cartilage that prefigures each future bone is transient and begins a process of maturation at the mid diaphysis in a well defined sequence of events involving hypertrophy and eventual replacement by bone (coordinated periosteal and endochondral ossification). This sequence of events progresses proximally and distally in each rudiment with growth plates persisting at the epiphyses to allow continued elongation. The site of the future synovial joint at the interface of the rudiments, is made up of three layers of cells that are histologically and molecularly distinct; the chondrogenous layers contouring the ends of the rudiments and the intervening intermediate layer where cavitation will later take place. Cell lineage marking of interzone cells in the mouse (expressing Gdf5) shows that they will give rise to all joint tissues including the articular cartilage and synovium with very little contribution to underlying epiphyseal bone[13], while marking of cells that express Matrilin 1 (within the rudiments) shows that they do not contribute to 
articular cartilage[14]. Therefore the cells that will give rise to transient cartilage in the rudiments and articular cartilage of the joint derive largely from distinct territories early in development (although there may be an intervening population of cells that can still contribute to both tissues). In contrast to transient cartilage, articular cartilage has a stable phenotype for the healthy lifetime of the joint with a unique striated 'zonal' architecture[15]. This tissue is vulnerable to breakdown with age and in disease states such as osteoarthritis (OA).

The musculoskeletal system develops in a co-ordinated fashion and this is seen clearly in the developing limb where muscle forms from cells that migrate into the limb bud, adjacent to the condensing mesenchyme cells that will form the cartilage template of the future skeleton[16]. This is also co-ordinated with the appearance of tendon specific markers in cells at the sites of muscle attachment to the rudiments and the gradual morphogenesis of functional tendons $[16,12]$. The forming muscle masses begin to contract precisely as the cartilage template is taking shape[12]. These closely associated developing tissues can therefore influence each other in a number of ways; through paracrine signalling between cell populations and through mechanical influences. While developing muscle is known to secrete paracrine signals[17] and signalling between developing tendon precursors and skeletal cells at the point of tendon attachment has been shown to be important in bone ridge initiation[18], direct evidence has also implicated mechanical forces generated by muscle contractions as necessary stimuli for the normal development of skeletal rudiments. Indications of this came from the described effects of congenital neuromuscular diseases, such as spinal muscular atrophy, which can cause partial or full intrauterine immobilization[19] leading to abnormal formation of long bones and susceptibility to fracture in the infant. Direct evidence came from animal models where the mechanical environment can be altered in a number of ways including in vivo immobilisation of the musculature or the use of mouse mutants where the skeletal rudiments develop with reduced, absent or non-contractile muscle (reviewed in[3]).

Similar findings in different animal models (chick, mouse, zebrafish), using either immobilization or absence/reduction of muscle, definitively separate paracrine and mechanical influences. Integrating these findings allows us to draw general conclusions about the specific aspects of skeletogenesis that require appropriate mechanical stimulation from adjacent twitching muscles for normal progression, including joint formation, ossification and rudiment shape (morphogenesis) (Figure 1). This underlines the importance of early movement for coordinated development of the whole musculoskeletal system. 


\section{Joint Formation:}

The accessibility of the chick embryo, where drug induced immobilization can be carried out with relative ease, allowed early demonstration of the particular sensitivity of joint formation to paralysis[20-24]. Immobilisation led to joint fusions in extreme cases with loss of cavitation, but also loss of associated structures such as articular surfaces, menisci and patella. This led to the general conclusion that positioning of the joints is not dependent on mechanical input from the muscle, but the relatively late event of cavitation is sensitive (reviewed in [11]). More recently, the use of several different mouse mutants where embryos

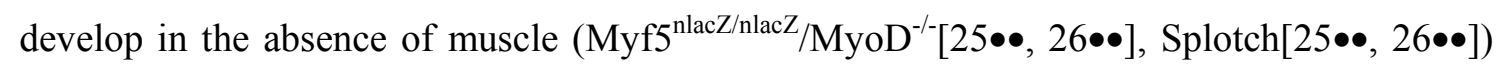
or reduced $\left(\mathrm{Myf}^{\text {nlacZ/+ }} / \mathrm{MyoD}^{-/}[27,25 \bullet \bullet]\right)$ or immobile muscle $\left(\mathrm{Mdg}^{-/-}[26 \bullet \bullet]\right.$ or Dock1 $1^{-/-[28]}$ (our unpublished results)), have shown similar joint fusions with particular sensitivity of the

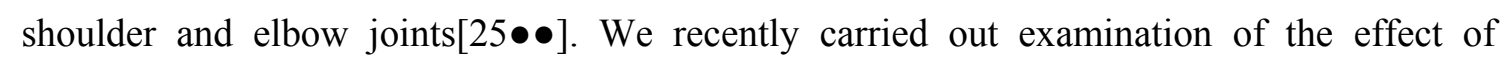
immobilization on development of the knee joint in the chick including 3D imaging of the developing region and the expression of genes that are either markers of emerging joint tissues or are known regulators of joint development [29••]. We also used 3D imaging to inform a computational model of muscle contractions in the developing knee region and showed correspondence between the predicted patterns of biophysical stimuli and the

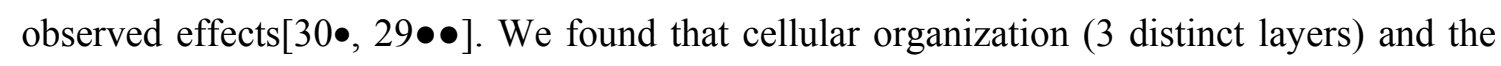
definition of tissue territories within the developing joint were lost, together with the expression of locally restricted regulatory molecules (e.g.BMP2, FGF2) as well as genes involved in hyaluronan function (HAS2 and CD44), implicated in the cavitation process. This shows that the cavitation process cannot be isolated as THE sensitive event and reflects more an impact on a continuous process of local patterning, defining multiple component tissues of the mature joint including articular cartilage (Figure 1B). We propose that the process of tissue definition requires appropriate mechanical stimuli which act as a form of positional information guiding position-specific differentiation. This is supported by muscleless mouse studies where cellular organization is also lost within the forming elbow and shoulder joints [25••] and where canonical Wnt signaling, necessary for the progressive development of the

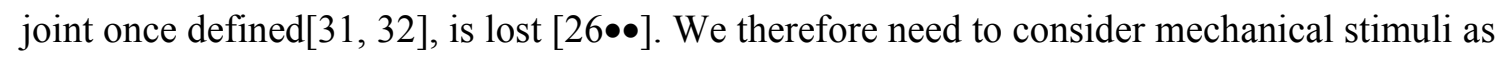
contributing to the spatial organization of location specific differentiation programmes rather than the cavitation process in isolation.

A notable difference between the effects seen in immobilized chick and mouse embryos is that forelimbs show greater sensitivity in the mouse whereas similar effects are 
seen in knee and elbow joints in the chick. Simulations of the biophysical stimuli produced by muscle contractions in both species and by passive movement (displacement of the limb), only relevant in the mouse where embryos develop in utero, offer a possible explanation for this[33••]. Due to the position, size and shape of the hindlimb, simulation of passive displacement predicted much higher stimuli impacting the rudiment tissues; this could compensate to some extent for the lack of self generated movement. However, this does not preclude other possible species specific differences in local regulatory mechanisms in fore and hindlimbs.

In addition to the loss of tissue territories, immobilization leads to changes in joint

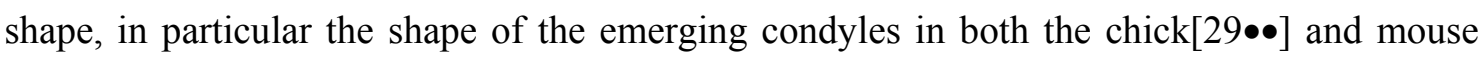
(see Morphogenesis below).

\section{Ossification:}

As described above, endochondral ossification begins with hypertrophy of chondrocytes at the mid- diaphysis of each long bone and the coordinated formation of an adjacent periosteal bone collar, progressing proximally and distally. Initial descriptions of $M y f 5 / M y o D$ double mutant, muscleless mouse embryos did not examine the ossification pattern at early stages of skeletogenesis but later stage rudiments(E18.5) showed alteration of size[34, 35], lack of fusion of palatal shelves[34] and effects on secondary ossification sites[36]. Sites of secondary cartilage in the avian jaw also depend on mechanical stimulation[37]. In the chick embryo we used computer simulations of embryonic muscle contractions to predict that peak biophysical stimuli (e.g. stress, strain, hydrostatic pressure) are experienced by cells at the mid-diaphysis prior to the initiation of ossification, spreading proximally and distally ahead of the wave of hypertrophy[38]. This led us to suggest a functional link between peak stimuli and maturation of chondrocytes toward hypertrophy/ ossification, corroborated by demonstrating reduced ossification at early stages of the process in immobilized embryos[39]. Therefore we felt it important to examine early stages of ossification in muscleless mouse embryos and showed that compared to stage-matched controls, ossification was reduced and abnormal in the scapula, humerus and femur[25••]. So although ossification proceeds, the altered pattern of initiation and progression shows that the process is not normal (Figure 1A). Gomez et al revealed increased cortical thickness of the femur at later stages[35] and more recently, Sharir et al (2011) used microCT of late stage long bones from non-

contractile muscle mutants $\left(\mathrm{Cacnas}^{\mathrm{mdg}} / \mathrm{Cacnas}^{\mathrm{mdg}}\right.$ ) to show that the normal asymmetric 
pattern of mineral deposition specific to each bone is lost in the absence of contractions,

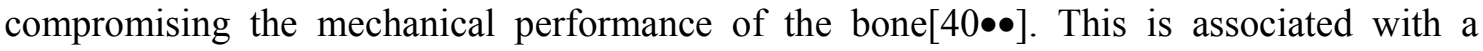
difference in osteoblast distribution. So muscle generated mechanical stimuli are required for normal initiation of ossification and normal local bone growth which reflects the hypomineralised and fragile bones seen in human infants with reduced uterine movement[19].

\section{Morphogenesis (rudiment shape):}

Each skeletal rudiment and joint of the limb can be identified by its unique, species specific, size and shape, appropriate to its contribution to a coordinated, refined system. Shape emerges through the local modulation of cellular processes, such as cell proliferation, differentiation, extracellular matrix synthesis, cell shape and size, but little is known about mechanisms that coordinate such processes to sculpt shape in the embryo. In the mature system, muscle loads affect precise bone shape, suggestive of co-ordinated evolution between muscle loads and the ability of the skeleton to bear the loads, and now evidence is accumulating from a number of different animal models and experimental immobilisations, that muscle loads also contribute to aspects of rudiment shape during development. 3D imaging of constituent tissues across developmental stages showed that the intricate shape of the chick knee joint emerges following the initiation of muscle contractions (HH28-34), with dramatic changes to typical protrusions (e.g. condyles) on the rudiment termini following the appearance of tendon markers at the points of muscle attachment (HH32-34), so that the knee joint at HH34 includes all the typical shape characteristics of the adult knee[12]. Elevated cell proliferation rates, corresponded to regions of higher dynamic stimuli predicted by Finite Element analysis and to the regions under relative expansion during development[30•]. We further showed that these typical characteristics are lost in immobilised embryos, corresponding with specific changes in spatial patterns of cell proliferation and altered gene expression patterns of specific tissue markers and regulatory genes including Pthrp which is known to control chondrocyte proliferation[29••]. Reduced chondrocyte proliferation was also recorded in growth plates of immobilised chick embryos[41].

In addition to affecting local patterns of cell proliferation, mechanical stimuli have also recently been implicated in the process of chondrocyte intercalation and column stacking important for rudiment elongation. In immobilized and muscleless zebrafish, pharyngeal cartilage morphology is abnormal with shorter elements and smaller and rounder 
chondrocytes lacking typical columnar organization[42••]. In mouse mutants with immobile muscle $(\mathrm{Spd})$, reduced rudiment length was not accompanied by lower cell numbers in growth plates, but a difference in cell shape was noted. Unlike in immobile zebrafish, cell columns did form but the columns were significantly shorter and it was suggested that the normal process, which combines short columns into elongated columns, does not take place[42••]. Although the molecular mechanisms driving chondrocyte intercalation are not fully known, the Wnt/PCP pathway[43, 44], and Wnt5a in particular[43], has been implicated in chondrocyte polarity and limb elongation.

\section{Integration of mechanical stimuli and molecular mechanisms: the mechanistic basis of a mechanoresponse}

The molecular mechanisms that control cartilage hypertrophy and ossification are relatively well defined[45-47], involving complex interactions between major signaling pathways; in particular a well described feedback loop between Ihh and Pthrp controls maturation to hypertrophy and the balance between ossification and elongation. Although control of joint development is comparatively less well understood, many of the same signaling pathways and role players have been implicated but with very distinct interactions and outcomes (reviewed in[15]). It is therefore interesting that both ossification and joint definition are altered when muscle contractions are absent or reduced[25]. It is clear that a balance of antichondrogenic and chondrogenic factors is needed in the joint and is likely required to maintain the stable phenotype of the articular cartilage, preventing maturation and hypertrophy; multiple alterations that tip the balance toward hypertrophy are associated with OA (reviewed in[15,48]. Early specification of the cells of the future joint is clearly important and many regulatory genes show restricted expression in these cells, however working out specific functions has been challenging. BMP signaling and its modulation through the antagonist Noggin is clearly important since Noggin inactivation leads to loss of joints[49]. Wnt signaling has also emerged as pivotal with very elegant gain of function and loss of function experiments showing it is required in particular to maintain the joint once specified[31, 32]; several assays show chondrogenic effects of BMP and antichondrogenic effects of Wnt activity.

The similarity of the effects seen when muscle is either absent or immobile and in both chick and mouse systems shows that common aspects of the phenotypes, on both ossification and joint development, are caused by altered mechanical stimuli. However we 
know nothing about how mechanical signals are integrated with other sources of positional cues and the cellular and molecular mechanisms that are implicated in differentiation and tissue patterning: This represents a major gap in our knowledge that is a challenge to address. Stretch Activated Ion Channels (SACs) have been implicated as one possible mechanism of mechanotransduction in chondrocytes[50] however, analysis of secondary cartilage induction in avian embryos shows that different mechanisms are at play in different sites and that SACs are not involved in secondary cartilage formation at the enthesis[37]. The characterisation of effects in muscleless mouse embryos in particular, establishes an experimental system that allows us to hone in on embryonic muscle contractions as the source of mechanical stimulation, and its impact on cellular differentiation and morphogenesis in developing skeletal rudiments and informs the collection of tissue samples (stimulated and unstimulated) for further analysis. We have carried out transcriptome analysis of rudiments under normal stimulation (control) and in the absence of limb muscle (Pax $\left.3^{\mathrm{Spd} / \mathrm{Spd}}\right)$ using both microarray and whole transcriptome sequencing (Rolfe et al., in preparation). Having defined that the earliest phenotypic impact was detected at Theiler stage (TS)23 (usually at embryonic day 14.5) at the outset of ossification and early patterning of tissue territories within the joint [25], and that the humerus and associated shoulder and elbow joints are the most severely affected, we chose this developmental time and tissue for analysis. Analysis of the differentially expressed (DE) gene sets using Gene Ontology terms showed strong specific enrichment of genes associated with development and differentiation, cytoskeletal organisation, ExtraCellular Matrix (ECM) and cell adhesion, providing important clues to the molecular mechanisms impacted by the altered stimuli. The DE gene sets also showed enormous enrichment for components of multiple cell signalling pathways with known roles in skeletal development (e.g. BMP, FGF, Notch, Hedgehog) and in particular Wnt signalling (Rolfe et al., in preparation). The molecular mechanisms impacted by mechanical stimuli in other developing systems (reviewed in[4,5]) might provide some clues to common mediators. Although much remains to be elucidated, it is clear that mechanical signals impact classic developmental signaling pathways including $\operatorname{Wnt}[51,7,8]$, can be sensed through cytoskeletal tension, possibly through integrins and deformation of other ECM components[52-55], and are often mediated by the Rho-Rock/ MyoII pathway[5].

In addition to providing insight into the mechanisms disturbed, this work also provides target genes and pathways for further analysis, to test hypotheses addressing molecular mechanisms involved in the mechanoresponse. Rot and Kablar (2013) have recently reported a microarray analysis of maxilla/palate complex tissues in control versus 
muscleless (Myf5:MyoD double nulls) E18.5 embryos[56] arising from their previous observation of cleft palate[34]. While this screen revealed genes associated with human congenital cleft palate, and also included genes encoding developmental regulators (signalling pathway components and transcription factors), there was very little overlap in the precise individual genes disregulated in these different developing systems.

\section{Response of progenitor cells in culture to mechanical stimuli: application to regenerative therapies}

A major challenge for the effective development of stem cell therapies for skeletal disorders is defining conditions required for priming of stable differentiation to produce appropriate tissues for repair and regeneration. As outlined above, cells that will form transient cartilage in the limb skeletal rudiment (will progress through hypertrophy and be replaced by bone) and cells at the rudiment termini that will form permanent articular cartilage, are already distinct early in development and experience different signalling environments, both biophysical and biochemical. Current attempts to differentiate adult derived stem cells in culture invariably induce transient chondrogenesis with progression to hypertrophy (e.g[57, 58]), a situation that is wholly unsatisfactory for articular repair and regeneration. A major outstanding developmental question is how articular cartilage progenitor cells are distinguished and then maintained in the embryo? It appears that even mature articular cartilage cells can be induced to undergo chondrogenic maturation and hypertrophy by treatment with 5-azacytidine[59]. Instability is also naturally seen with age and in OA. The specific mechanical environment of the developing and mature joint contribute to the maintenance of the stable phenotype as altering mechanical stimulation leads to loss of articular territories[29••]. Understanding the basis of this maintenance and the contribution of mechanical stimuli would be a major step forward in development of new approaches to promote stable chondrogenesis of mesenchymal stem cells (MSCs), resistant to hypertrophy. Conversely, this knowledge could also be critical in enabling improved endochondral bone regeneration[60]. The concept of applying knowledge gained from the developing embryo to in vitro priming of stem cells for regenerative therapies is fundamental and is gaining attention in the field of tissue engineering (reviewed in[61, 62])

A wealth of evidence shows that various mechanical stimuli influence the differentiation of progenitor cells isolated from adult sources (reviewed in[63, 64]). These studies demonstrate the importance of mechanical stimuli but have varied results depending 
on cell context[63] - for example compression can enhance chondrogenesis in MSCs but it depends on when the load is applied and in what cellular environment[65-67]. From a developmental perspective this is not surprising:- context dependent responses are a common feature of development. Part of the variability is likely to be due to the issue of heterogeneity among MSC populations from different sources[68] and under different purification protocols; optimal cell sources and purification strategies need to be defined for specific tissue regeneration approaches, requiring extensive analysis to investigate cell responses[62]. Again knowledge gained from the developing embryo would be important in defining appropriate markers to assess cellular response. This will be required before any therapy can enter the clinic.

Despite variability in response, some generalities can be drawn from the work on biophysical stimulation of stem cells to date. The computational model generated by Prendergast et al (1997) proposed that a balance of fluid flow and shear strain determines the spatial and temporal pattern of MSC cell fate and this prediction has been corroborated by various experimental results including work in bone chambers or 3D scaffolds[69, 70]. Hydrostatic Pressure (HP) has been largely associated with chondrogenic differentiation whereas tensile strain and fluid induced shear stress are generally shown to induce osteogenesis[71]. HP, which occurs during loading of the joint, is predicted by finite element modeling to promote cartilage formation and suppress endochondral ossification[73-74]. Experimentally cyclic HP applied to MSCs in defined medium was found to enhance chondrogenesis, increasing expression of chondrogenic markers[75-77]. Several parameters, including magnitude, frequency, onset and duration of HP stimuli are important in the response[78-80]. Application of HP has recently been shown to increase the functional properties of cartilageous tissues[81] and to synergise with TGF $\beta$ signalling in generating a more stable chondrogenic phenotype with reduced levels of hypertrophic markers[82].

Cyclic tensile strain is prominent in the superficial region of cartilage tissue and at the cartilage-bone interface and is predicted (with octahedral shear stress) to stimulate maturation and ossification[72] and is experimentally associated with enhanced osteogenic differentiation of stem cells. Tensile strain applied in a two-dimensional culture caused a switch in cell fate from adipogenic to osteogenic differentiation via up-regulation of canonical Wnt responsive genes[83]. Wnt signalling (through $\beta$-catenin) was also implicated in the osteogenic differentiation of bone marrow derived cells embedded in alginate-gels exposed to dynamic tension[84]. Similarily short-term cyclic tensile strain reduced the rate of 
MSC proliferation and induced osteogenic differentiation[85]. Conversely, Connolly et al. (2010) found that cyclic tensile loading of bone marrow stromal cells promoted fibrocartilage-like differentiation[86]. Fluid-induced shear stress has also been shown to promote osteogenic differentiation[87-89] through RhoA/ROCK2 and enhanced tension in the actin cytoskeleton[88]. In a similar study oscillatory fluid-flow induced $\beta$-catenin nuclear translocation and up-regulation of Wnt associated proteins, Wnt5a and Ror2, which are both involved in RhoA activation[87] and ultimately Runx2 expression[88]. A significant decrease in $\beta$-catenin $/ \mathrm{N}$-cadherin association following fluid-flow was also shown, resulting in an increase in cytoplasmic $\beta$-catenin[87], suggesting a role for adherens junctions as mechanosensors. In three-dimensional cultures a continuous level of flow-induced shear stress showed an increase in collagen synthesis, and an increase in the tensile mechanical properties of the tissue[90]. Application of fluid-flow perfusion increased ECM deposition compared to static control, demonstrating a potential beneficial effect also on chondrogenic differentiation[91].

Our work modeling biophysical stimuli generated by muscle contractions in developing skeletal rudiments predicted a number of interesting patterns corresponding to differentiation events, corroborated by phenotypic effects in abnormal mechanical environments: 1) a sustained period of cyclic stress at the perichondrium promotes chondrocyte hypertrophy at the core, stimulating subsequent bone collar formation[38]; 2) Modelling of stimuli in the joint region indicated that the articular cartilages and patella develop under the influence of very specific patterns of biophysical stimuli; the chondrogenous layers emerge from locations that experience distinct patterns of elevated fluid flow while the patella develops under much higher magnitudes of stress, fluid velocity and pore pressure[30•].

\section{Conclusion}

In order to effectively apply biophysical stimuli to cellular differentiation regimes for regenerative therapies, we need to know more about how cells in the developing embryo respond, in different contexts, across space and time as the tissues emerge. The accumulating evidence reviewed here shows that several aspects of normal skeletogenesis require appropriate mechanical stimulation generated by embryonic movement; tissue patterning during joint formation, ossification and rudiment morphogenesis (Figure 1). We now need to discover the precise molecular events guided by mechanical stimuli and how mechanical and biochemical signals are integrated in order to apply this knowledge in therapeutic approaches. Clearly the effects of biophysical stimuli will depend on the cell context and other aspects of 
the signalling - mileau. Therefore more testing is needed on defined populations of progenitor cells to reproduce the integrated biochemical and mechanical signalling environment[82]. Bioreactor systems could be developed to reproduce multiple aspects of in vivo developmental conditions aimed at creating tissues with improved and more stable functional properties. It would be unrealistic to expect to reproduce all conditions that guide normal development of, for example, articular cartilage, however improved knowledge of the integrated mechanisms and their outcome, combined with tailor made bioreactor systems would allow definition of essential differentiation regimes toward establishing regenerative therapies for diseased or aged joints.

1. Frost HM. The mechanostat: a proposed pathogenic mechanism of osteoporoses and the bone mass effects of mechanical and nonmechanical agents. Bone and mineral. 1987;2(2):7385.

2. Frost HM. The Utah paradigm of skeletal physiology: an overview of its insights for bone, cartilage and collagenous tissue organs. J Bone Miner Metab. 2000;18(6):305-16.

3. Nowlan NC, Sharpe J, Roddy KA, Prendergast PJ, Murphy P. Mechanobiology of embryonic skeletal development: Insights from animal models. Birth Defects Res C Embryo Today. 2010;90(3):203-13. doi:10.1002/bdrc.20184 [doi].

4. Farge E. Mechanotransduction in development. Curr Top Dev Biol. 2011;95:243-65. doi:10.1016/b978-0-12-385065-2.00008-6.

5. Mammoto A, Mammoto T, Ingber DE. Mechanosensitive mechanisms in transcriptional regulation. J Cell Sci. 2012;125(Pt 13):3061-73. doi:10.1242/jcs.093005.

-6. Mammoto T, Mammoto A, Torisawa YS, Tat T, Gibbs A, Derda $\mathrm{R}$ et al. Mechanochemical control of mesenchymal condensation and embryonic tooth organ formation. Dev Cell. 2011;21(4):758-69. doi:10.1016/j.devcel.2011.07.006. A key paper in advancing our understanding of how mechanical changes are integrated with molecular mechanisms of development. Demonstrates that change in cell shape (cytoskeleton) is a necessary step in signalling from ectoderm to mesoderm and induction of tooth bud formation.

7. Aigouy B, Farhadifar R, Staple DB, Sagner A, Roper JC, Julicher F et al. Cell flow reorients the axis of planar polarity in the wing epithelium of Drosophila. Cell. 2010;142(5):773-86. doi:10.1016/j.cell.2010.07.042. 
8. Olguin P, Glavic A, Mlodzik M. Intertissue mechanical stress affects Frizzled-mediated planar cell polarity in the Drosophila notum epidermis. Curr Biol. 2011;21(3):236-42. doi:10.1016/j.cub.2011.01.001.

9. Hinchliffe JR, Johnson DR. The development of the vertebrate limb In: Limb development in tetrapods. 1980:72-83.

10. Pacifici M, Koyama E, Iwamoto M. Mechanisms of synovial joint and articular cartilage formation: recent advances, but many lingering mysteries. Birth Defects Res C Embryo Today. 2005;75(3):237-48. doi:10.1002/bdrc.20050.

11. Pitsillides AA, Ashhurst DE. A critical evaluation of specific aspects of joint development. Dev Dyn. 2008;237(9):2284-94. doi:10.1002/dvdy.21654.

12. Roddy KA, Nowlan NC, Prendergast PJ, Murphy P. 3D representation of the developing chick knee joint: a novel approach integrating multiple components. J Anat. 2009;214(3):374-87. doi:JOA1040 [pii]

10.1111/j.1469-7580.2008.01040.x [doi].

13. Koyama E, Shibukawa Y, Nagayama M, Sugito H, Young B, Yuasa T et al. A distinct cohort of progenitor cells participates in synovial joint and articular cartilage formation during mouse limb skeletogenesis. Dev Biol. 2008;316(1):62-73. doi:10.1016/j.ydbio.2008.01.012.

14. Hyde G, Dover S, Aszodi A, Wallis GA, Boot-Handford RP. Lineage tracing using matrilin-1 gene expression reveals that articular chondrocytes exist as the joint interzone forms. Dev Biol. 2007;304(2):825-33. doi:10.1016/j.ydbio.2007.01.026.

15. Onyekwelu I, Goldring MB, Hidaka C. Chondrogenesis, joint formation, and articular cartilage regeneration. J Cell Biochem. 2009;107(3):383-92. doi:10.1002/jcb.22149.

16. Kardon G. Muscle and tendon morphogenesis in the avian hind limb. Development. 1998;125(20):4019-32.

17. Henningsen J, Rigbolt KT, Blagoev B, Pedersen BK, Kratchmarova I. Dynamics of the skeletal muscle secretome during myoblast differentiation. Mol Cell Proteomics. 2010;9(11):2482-96. doi:10.1074/mcp.M110.002113.

18. Blitz E, Viukov S, Sharir A, Shwartz Y, Galloway JL, Pryce BA et al. Bone ridge patterning during musculoskeletal assembly is mediated through SCX regulation of Bmp4 at the tendon-skeleton junction. Dev Cell. 2009;17(6):861-73. doi:10.1016/j.devcel.2009.10.010. 
19. Rodriguez JI, Palacios J, Garcia-Alix A, Pastor I, Paniagua R. Effects of immobilization on fetal bone development. A morphometric study in newborns with congenital neuromuscular diseases with intrauterine onset. Calcif Tissue Int. 1988;43(6):335-9.

20. Drachman DBS, L. The role of movement in embryonic joint development. Developmental Biology. 1966;14:401-20.

21. Murray PD, Drachman DB. The role of movement in the development of joints and related structures: the head and neck in the chick embryo. J Embryol Exp Morphol. 1969;22(3):349-71.

22. Osborne AC, Lamb KJ, Lewthwaite JC, Dowthwaite GP, Pitsillides AA. Short-term rigid and flaccid paralyses diminish growth of embryonic chick limbs and abrogate joint cavity formation but differentially preserve pre-cavitated joints. J Musculoskelet Neuronal Interact. 2002;2(5):448-56.

23. Persson M. The role of movements in the development of sutural and diarthrodial joints tested by long-term paralysis of chick embryos. J Anat. 1983;137 (Pt 3):591-9.

24. Ruano-Gil D, Nardi-Vilardaga J, Tejedo-Mateu A. Influence of extrinsic factors on the development of the articular system. Acta Anat (Basel). 1978;101(1):36-44.

••25. Nowlan NC, Bourdon C, Dumas G, Tajbakhsh S, Prendergast PJ, Murphy P. Developing bones are differentially affected by compromised skeletal muscle formation. Bone. 2010;46(5):1275-85. doi:S8756-3282(09)02057-2 [pii] 10.1016/j.bone.2009.11.026 [doi]. Establishes that mouse embryos developing in the absence of muscle contractions (Muscleless and reduced muscle) have abnormal ossification, joint formation and morphogenesis of rudiment shape.

••26. Kahn J, Shwartz Y, Blitz E, Krief S, Sharir A, Breitel DA et al. Muscle contraction is necessary to maintain joint progenitor cell fate. Dev Cell. 2009;16(5):734-43. doi:10.1016/j.devcel.2009.04.013. Describes the effects of lack of muscle and immobile muscle on forming joints. Demonstrates that joint formation fails in particular sites and that the canonical Wht pathway is implicated in the repsonse to muscle contractions.

27. Rudnicki MA, Schnegelsberg PN, Stead RH, Braun T, Arnold HH, Jaenisch R. MyoD or Myf-5 is required for the formation of skeletal muscle. Cell. 1993;75(7):1351-9. doi:00928674(93)90621-V [pii].

28. Laurin M, Fradet N, Blangy A, Hall A, Vuori K, Cote JF. The atypical Rac activator Dock180 (Dock1) regulates myoblast fusion in vivo. Proc Natl Acad Sci U S A. 2008;105(40):15446-51. doi:10.1073/pnas.0805546105. 
$\bullet \bullet 29$. Roddy KA, Prendergast PJ, Murphy P. Mechanical influences on morphogenesis of the knee joint revealed through morphological, molecular and computational analysis of immobilised embryos. PLoS One. 2011;6(2):e17526. doi:10.1371/journal.pone.0017526. Chick embryos immobilised in ovo by a neuromuscular blocking agent were analysed, specifically to reveal impacts on knee joint formation. 3D imaging and gene expression analysis revealed that organised tissue territories in the forming joint region are lost and the morphogenesis of rudiment shape is affected.

•30. Roddy KA, Kelly GM, van Es MH, Murphy P, Prendergast PJ. Dynamic patterns of mechanical stimulation co-localise with growth and cell proliferation during morphogenesis in the avian embryonic knee joint. J Biomech. 2011. doi:S0021-9290(10)00486-0 [pii] 10.1016/j.jbiomech.2010.08.039 [doi]. A Finite Element simulation of a flexion-extension contraction cycle at the knee joint was informed by 3D imaging of deveolping embryo limbs and predicted the biophysical stimuli generated in different regions of the presumptive joint over time.

31. Guo X, Day TF, Jiang X, Garrett-Beal L, Topol L, Yang Y. Wnt/beta-catenin signaling is sufficient and necessary for synovial joint formation. Genes Dev. 2004;18(19):2404-17. doi:10.1101/gad.1230704.

32. Spater D, Hill TP, O'Sullivan R J, Gruber M, Conner DA, Hartmann C. Wnt9a signaling is required for joint integrity and regulation of Ihh during chondrogenesis. Development. 2006;133(15):3039-49. doi:10.1242/dev.02471.

••33. Nowlan NC, Dumas G, Tajbakhsh S, Prendergast PJ, Murphy P. Biophysical stimuli induced by passive movements compensate for lack of skeletal muscle during embryonic skeletogenesis. Biomech Model Mechanobiol. 2012;11(1-2):207-19. doi:10.1007/s10237011-0304-4. Finite Element Analysis suggested a possible explanation for the observed increased impact on the forelimb compared to hindlimb when mechanical stimulation from muscle contractions is absent; it predicted that passive movement generates greater stimulation of the hindlimb. 34. Rot-Nikcevic I, Reddy T, Downing KJ, Belliveau AC, HallgrÃ-msson B, Hall BK et al. Myf5-/-:MyoD-/- amyogenic fetuses reveal the importance of early contraction and static loading by striated muscle in mouse skeletogenesis. Development Genes and Evolution. 2006;216(1):1-9.

35. Gomez C, David V, Peet NM, Vico L, Chenu C, Malaval L et al. Absence of mechanical loading in utero influences bone mass and architecture but not innervation in Myod-Myf5- 
deficient mice. J Anat. 2007;210(3):259-71. doi:JOA698 [pii] 10.1111/j.14697580.2007.00698.x [doi].

36. Rot-Nikcevic I, Downing KJ, Hall BK, Kablar B. Development of the mouse mandibles and clavicles in the absence of skeletal myogenesis. Histology and histopathology. 2007;22(1):51-60.

37. Solem RC, Eames BF, Tokita M, Schneider RA. Mesenchymal and mechanical mechanisms of secondary cartilage induction. Dev Biol. 2011;356(1):28-39. doi:10.1016/j.ydbio.2011.05.003.

38. Nowlan NC, Murphy P, Prendergast PJ. A dynamic pattern of mechanical stimulation promotes ossification in avian embryonic long bones. J Biomech. 2008;41(2):249-58. doi:S0021-9290(07)00397-1 [pii] 10.1016/j.jbiomech.2007.09.031 [doi].

39. Nowlan NC, Prendergast PJ, Murphy P. Identification of mechanosensitive genes during embryonic bone formation. PLoS Comput Biol. 2008;4(12):e1000250. doi:10.1371/journal.pcbi.1000250 [doi].

$\bullet \bullet 40$. Sharir A, Stern T, Rot C, Shahar R, Zelzer E. Muscle force regulates bone shaping for optimal load-bearing capacity during embryogenesis. Development. 2011;138(15):3247-59. doi:10.1242/dev.063768. Evidence that the removal of muscle contractions leads to a change in the normal asymmetric pattern of bone mineral deposition and to mechanically inferior bones

41. Germiller JA, Goldstein SA. Structure and function of embryonic growth plate in the absence of functioning skeletal muscle. Journal of orthopaedic research : official publication of the Orthopaedic Research Society. 1997;15(3):362-70. doi:10.1002/jor.1100150308.

••42. Shwartz Y, Farkas Z, Stern T, Aszodi A, Zelzer E. Muscle contraction controls skeletal morphogenesis through regulation of chondrocyte convergent extension. Dev Biol. 2012;370(1):154-63. doi:10.1016/j.ydbio.2012.07.026. Evidence for the role of mechanical stimulation from muscle contraction in zebrafish skeletal development. Reveals an impact on chondrocyte shape and columnar organisation in zebrafish and on chondrocte intercalation in the growth plate of muscle-less mouse embryos.

43. Gao B, Song H, Bishop K, Elliot G, Garrett L, English MA et al. Wnt signaling gradients establish planar cell polarity by inducing Vang12 phosphorylation through Ror2. Dev Cell. 2011;20(2):163-76. doi:10.1016/j.devcel.2011.01.001.

44. Li Y, Dudley AT. Noncanonical frizzled signaling regulates cell polarity of growth plate chondrocytes. Development. 2009;136(7):1083-92. doi:10.1242/dev.023820. 
45. Kronenberg HM. Developmental regulation of the growth plate. Nature. 2003;423(6937):332-6.

46. Provot S, Schipani E. Molecular mechanisms of endochondral bone development. Biochem Biophys Res Commun. 2005;328(3):658-65. doi:S0006-291X(04)02642-7 [pii] 10.1016/j.bbrc.2004.11.068 [doi].

47. Studer D, Millan C, Ozturk E, Maniura-Weber K, Zenobi-Wong M. Molecular and biophysical mechanisms regulating hypertrophic differentiation in chondrocytes and mesenchymal stem cells. Eur Cell Mater. 2012;24:118-35; discussion 35.

48. Pitsillides AA, Beier F. Cartilage biology in osteoarthritis--lessons from developmental biology. Nat Rev Rheumatol. 2011;7(11):654-63. doi:10.1038/nrrheum.2011.129.

49. Brunet LJ, McMahon JA, McMahon AP, Harland RM. Noggin, cartilage morphogenesis, and joint formation in the mammalian skeleton. Science. 1998;280(5368):1455-7.

50. $\mathrm{Wu} \mathrm{QQ}$, Chen Q. Mechanoregulation of chondrocyte proliferation, maturation, and hypertrophy: ion-channel dependent transduction of matrix deformation signals. Experimental cell research. 2000;256(2):383-91. doi:10.1006/excr.2000.4847.

51. Desprat N, Supatto W, Pouille PA, Beaurepaire E, Farge E. Tissue deformation modulates twist expression to determine anterior midgut differentiation in Drosophila embryos. Dev Cell. 2008;15(3):470-7. doi:10.1016/j.devcel.2008.07.009.

52. Geiger B, Spatz JP, Bershadsky AD. Environmental sensing through focal adhesions. Nat Rev Mol Cell Biol. 2009;10(1):21-33. doi:10.1038/nrm2593.

53. Chowdhury F, Na S, Li D, Poh YC, Tanaka TS, Wang F et al. Material properties of the cell dictate stress-induced spreading and differentiation in embryonic stem cells. Nature materials. 2010;9(1):82-8. doi:10.1038/nmat2563.

54. del Rio A, Perez-Jimenez R, Liu R, Roca-Cusachs P, Fernandez JM, Sheetz MP. Stretching single talin rod molecules activates vinculin binding. Science. 2009;323(5914):638-41. doi:10.1126/science.1162912.

55. Sansores-Garcia L, Bossuyt W, Wada K, Yonemura S, Tao C, Sasaki H et al. Modulating F-actin organization induces organ growth by affecting the Hippo pathway. EMBO J. 2011;30(12):2325-35. doi:10.1038/emboj.2011.157.

56. Rot I, Kablar B. Role of skeletal muscle in palate development. Histol Histopathol. 2013;28(1):1-13.

57. Mueller MB, Fischer M, Zellner J, Berner A, Dienstknecht T, Prantl L et al. Hypertrophy in mesenchymal stem cell chondrogenesis: effect of TGF-beta isoforms and chondrogenic conditioning. Cells, tissues, organs. 2010;192(3):158-66. doi:10.1159/000313399. 
58. Dickhut A, Pelttari K, Janicki P, Wagner W, Eckstein V, Egermann M et al. Calcification or dedifferentiation: requirement to lock mesenchymal stem cells in a desired differentiation stage. J Cell Physiol. 2009;219(1):219-26. doi:10.1002/jcp.21673.

59. Zuscik MJ, Baden JF, Wu Q, Sheu TJ, Schwarz EM, Drissi H et al. 5-azacytidine alters TGF-beta and BMP signaling and induces maturation in articular chondrocytes. Journal of cellular biochemistry. 2004;92(2):316-31. doi:10.1002/jcb.20050.

60. Farrell E, Both SK, Odorfer KI, Koevoet W, Kops N, O'Brien FJ et al. In-vivo generation of bone via endochondral ossification by in-vitro chondrogenic priming of adult human and rat mesenchymal stem cells. BMC musculoskeletal disorders. 2011;12:31. doi:10.1186/14712474-12-31.

61. Lenas P, Moos M, Luyten FP. Developmental engineering: a new paradigm for the design and manufacturing of cell-based products. Part I: from three-dimensional cell growth to biomimetics of in vivo development. Tissue engineering Part B, Reviews. 2009;15(4):381-94. doi:10.1089/ten.TEB.2008.0575.

62. Hellingman CA, Koevoet W, van Osch GJ. Can one generate stable hyaline cartilage from adult mesenchymal stem cells? A developmental approach. Journal of tissue engineering and regenerative medicine. 2012;6(10):e1-e11. doi:10.1002/term.502.

63. Kelly DJ, Jacobs CR. The role of mechanical signals in regulating chondrogenesis and osteogenesis of mesenchymal stem cells. Birth Defects Research Part C: Embryo Today: Reviews. 2010;90(1):75-85.

64. Responte DJ, Lee JK, Hu JC, Athanasiou KA. Biomechanics-driven chondrogenesis: from embryo to adult. FASEB J. 2012;26(9):3614-24. doi:10.1096/fj.12-207241.

65. Angele P, Schumann D, Angele M, Kinner B, Englert C, Hente R et al. Cyclic, mechanical compression enhances chondrogenesis of mesenchymal progenitor cells in tissue engineering scaffolds. Biorheology. 2004;41(3-4):335-46.

66. Mouw JK, Connelly JT, Wilson CG, Michael KE, Levenston ME. Dynamic compression regulates the expression and synthesis of chondrocyte-specific matrix molecules in bone marrow stromal cells. Stem Cells. 2007;25(3):655-63. doi:10.1634/stemcells.2006-0435.

67. Thorpe SD, Buckley CT, Vinardell T, O'Brien FJ, Campbell VA, Kelly DJ. Dynamic compression can inhibit chondrogenesis of mesenchymal stem cells. Biochem Biophys Res Commun. 2008;377(2):458-62. doi:S0006-291X(08)01946-3 [pii]

10.1016/j.bbrc.2008.09.154 [doi].

68. Phinney DG. Functional heterogeneity of mesenchymal stem cells: implications for cell therapy. Journal of cellular biochemistry. 2012;113(9):2806-12. doi:10.1002/jcb.24166. 
69. McMahon LA, Reid AJ, Campbell VA, Prendergast PJ. Regulatory effects of mechanical strain on the chondrogenic differentiation of MSCs in a collagen-GAG scaffold: experimental and computational analysis. Ann Biomed Eng. 2008;36(2):185-94. doi:10.1007/s10439-0079416-5.

70. Khayyeri H, Checa S, Tagil M, Prendergast PJ. Corroboration of mechanobiological simulations of tissue differentiation in an in vivo bone chamber using a lattice-modeling approach. Journal of orthopaedic research : official publication of the Orthopaedic Research Society. 2009;27(12):1659-66. doi:10.1002/jor.20926.

71. Carter DR, Orr TE, Fyhrie DP, Schurman DJ. Influences of mechanical stress on prenatal and postnatal skeletal development. Clin Orthop Relat Res. 1987(219):237-50.

72. Carter DR, Wong M. Modelling cartilage mechanobiology. Philos Trans R Soc Lond B Biol Sci. 2003;358(1437):1461-71. doi:10.1098/rstb.2003.1346.

73. Carter DR, Beaupre GS, Giori NJ, Helms JA. Mechanobiology of skeletal regeneration. Clinical orthopaedics and related research. 1998(355 Suppl):S41-55.

74. Loboa EG, Beaupre GS, Carter DR. Mechanobiology of initial pseudarthrosis formation with oblique fractures. J Orthop Res. 2001;19(6):1067-72. doi:10.1016/s07360266(01)00028-6.

75. Angele P, Yoo JU, Smith C, Mansour J, Jepsen KJ, Nerlich M et al. Cyclic hydrostatic pressure enhances the chondrogenic phenotype of human mesenchymal progenitor cells differentiated in vitro. J Orthop Res. 2003;21(3):451-7. doi:10.1016/s0736-0266(02)00230-9. 76. Miyanishi K, Trindade MC, Lindsey DP, Beaupre GS, Carter DR, Goodman SB et al. Effects of hydrostatic pressure and transforming growth factor-beta 3 on adult human mesenchymal stem cell chondrogenesis in vitro. Tissue Eng. 2006;12(6):1419-28. doi:10.1089/ten.2006.12.1419 [doi].

77. Wagner DR, Lindsey DP, Li KW, Tummala P, Chandran SE, Smith RL et al. Hydrostatic pressure enhances chondrogenic differentiation of human bone marrow stromal cells in osteochondrogenic medium. Ann Biomed Eng. 2008;36(5):813-20. doi:10.1007/s10439-0089448-5.

78. Elder SH, Fulzele KS, McCulley WR. Cyclic hydrostatic compression stimulates chondroinduction of C3H/10T1/2 cells. Biomech Model Mechanobiol. 2005;3(3):141-6. doi:10.1007/s10237-004-0058-3 [doi].

79. Ikenoue T, Trindade MC, Lee MS, Lin EY, Schurman DJ, Goodman SB et al. Mechanoregulation of human articular chondrocyte aggrecan and type II collagen expression 
by intermittent hydrostatic pressure in vitro. J Orthop Res. 2003;21(1):110-6. doi:10.1016/s0736-0266(02)00091-8.

80. Miyanishi K, Trindade MC, Lindsey DP, Beaupre GS, Carter DR, Goodman SB et al. Dose- and time-dependent effects of cyclic hydrostatic pressure on transforming growth factor-beta3-induced chondrogenesis by adult human mesenchymal stem cells in vitro. Tissue Eng. 2006;12(8):2253-62. doi:10.1089/ten.2006.12.2253 [doi].

81. Meyer EG, Buckley CT, Steward AJ, Kelly DJ. The effect of cyclic hydrostatic pressure on the functional development of cartilaginous tissues engineered using bone marrow derived mesenchymal stem cells. J Mech Behav Biomed Mater. 2011;4(7):1257-65. doi:10.1016/j.jmbbm.2011.04.012.

82. Vinardell T, Rolfe RA, Buckley CT, Meyer EG, Ahearne M, Murphy P et al. Hydrostatic pressure acts to stabilise a chondrogenic phenotype in porcine joint tissue derived stem cells. Eur Cell Mater. 2012;23:121-32; discussion 33-4.

83. Sen B, Xie Z, Case N, Ma M, Rubin C, Rubin J. Mechanical strain inhibits adipogenesis in mesenchymal stem cells by stimulating a durable beta-catenin signal. Endocrinology. 2008;149(12):6065-75. doi:10.1210/en.2008-0687.

84. Haudenschild AK, Hsieh AH, Kapila S, Lotz JC. Pressure and distortion regulate human mesenchymal stem cell gene expression. Ann Biomed Eng. 2009;37(3):492-502. doi:10.1007/s10439-008-9629-2.

85. Kearney EM, Farrell E, Prendergast PJ, Campbell VA. Tensile strain as a regulator of mesenchymal stem cell osteogenesis. Ann Biomed Eng. 2010;38(5):1767-79. doi:10.1007/s10439-010-9979-4.

86. Connelly JT, Vanderploeg EJ, Mouw JK, Wilson CG, Levenston ME. Tensile loading modulates bone marrow stromal cell differentiation and the development of engineered fibrocartilage constructs. Tissue Eng Part A. 2010;16(6):1913-23. doi:10.1089/ten.TEA.2009.0561.

87. Arnsdorf EJ, Tummala P, Jacobs CR. Non-canonical Wnt signaling and N-cadherin related beta-catenin signaling play a role in mechanically induced osteogenic cell fate. PLoS One. 2009;4(4):e5388. doi:10.1371/journal.pone.0005388.

88. Arnsdorf EJ, Tummala P, Kwon RY, Jacobs CR. Mechanically induced osteogenic differentiation--the role of RhoA, ROCKII and cytoskeletal dynamics. J Cell Sci. 2009;122(Pt 4):546-53. doi:10.1242/jcs.036293. 
89. Sharp LA, Lee YW, Goldstein AS. Effect of low-frequency pulsatile flow on expression of osteoblastic genes by bone marrow stromal cells. Ann Biomed Eng. 2009;37(3):445-53. doi:10.1007/s 10439-008-9632-7.

90. Gemmiti CV, Guldberg RE. Shear stress magnitude and duration modulates matrix composition and tensile mechanical properties in engineered cartilaginous tissue. Biotechnol Bioeng. 2009;104(4):809-20. doi:10.1002/bit.22440.

91. Alves da Silva ML, Martins A, Costa-Pinto AR, Correlo VM, Sol P, Bhattacharya M et al. Chondrogenic differentiation of human bone marrow mesenchymal stem cells in chitosanbased scaffolds using a flow-perfusion bioreactor. J Tissue Eng Regen Med. 2011;5(9):72232. doi:10.1002/term.372.

92. Kim IS, Otto F, Zabel B, Mundlos S. Regulation of chondrocyte differentiation by Cbfa1. Mech Dev. 1999;80(2):159-70.

\section{Figure Legend:}

Figure 1: Lack of mechanical stimulation from muscle contractions impacts several aspects of skeletal development: the mouse forelimb and chick hindlimb are used here to summarise the effects on ( $\left(\frac{\cdots}{*}\right)$ ossification [e.g. 25, 38, 39], (A) joint formation [e.g. 25, 26, 29, 23] and ( $\boldsymbol{V})$ rudiment shape (Morphogenesis) [e.g. 25, 29, 40, 42, 34,35, 41]. Mouse embryos show strongest (but not exclusive) effects on the rudiments and joints of the forelimb. The data shown in boxes a, b and c serve as examples of the evidence of impact on each process. a) mid diaphysis of the mouse humerus at TS23 (E14.5) in control and Spd mutant (muscleless), stage matched embryos, showing expression of Spp1. The Spp1 gene is normally expressed in the hypertrophic zone [92]. Despite the appearance of hypertrophic chondrocytes at the mid-diaphysis of immobile Spd embryos, no Spp1 gene expression is detected (our unpublished results). b) In the knee joint of immobilised chick embryos, lack of definition of joint territories is shown by histology (note the absence of organised cell layers within the presumptive joint) and gene expression patterns (as indicated) which normally define the territories of the forming joint; the periarticular cartilage of the rudiment (PthrP), the intermediate layer of the interzone (Bmp2). The schematic summarises effects of immobilisation on the knee joint. c) The effect on rudiment morphogenesis is exemplified by the change in shape of the distal femur; the diagram represents outlines of control (blue) and immobilised (red) sections, overlaid (plane of section indicated). Note the change in height of the condyles (black brackets), narrowing of the intercondylar fossa (coloured brackets), and 
reduction in outgrowth of typical condylar spurs (arrow heads), particularly at the interface with the fibula (tf troclea fibularis groove). Images in b and c adapted from Roddy et al., 2011[29]. 
Mouse Forelimb

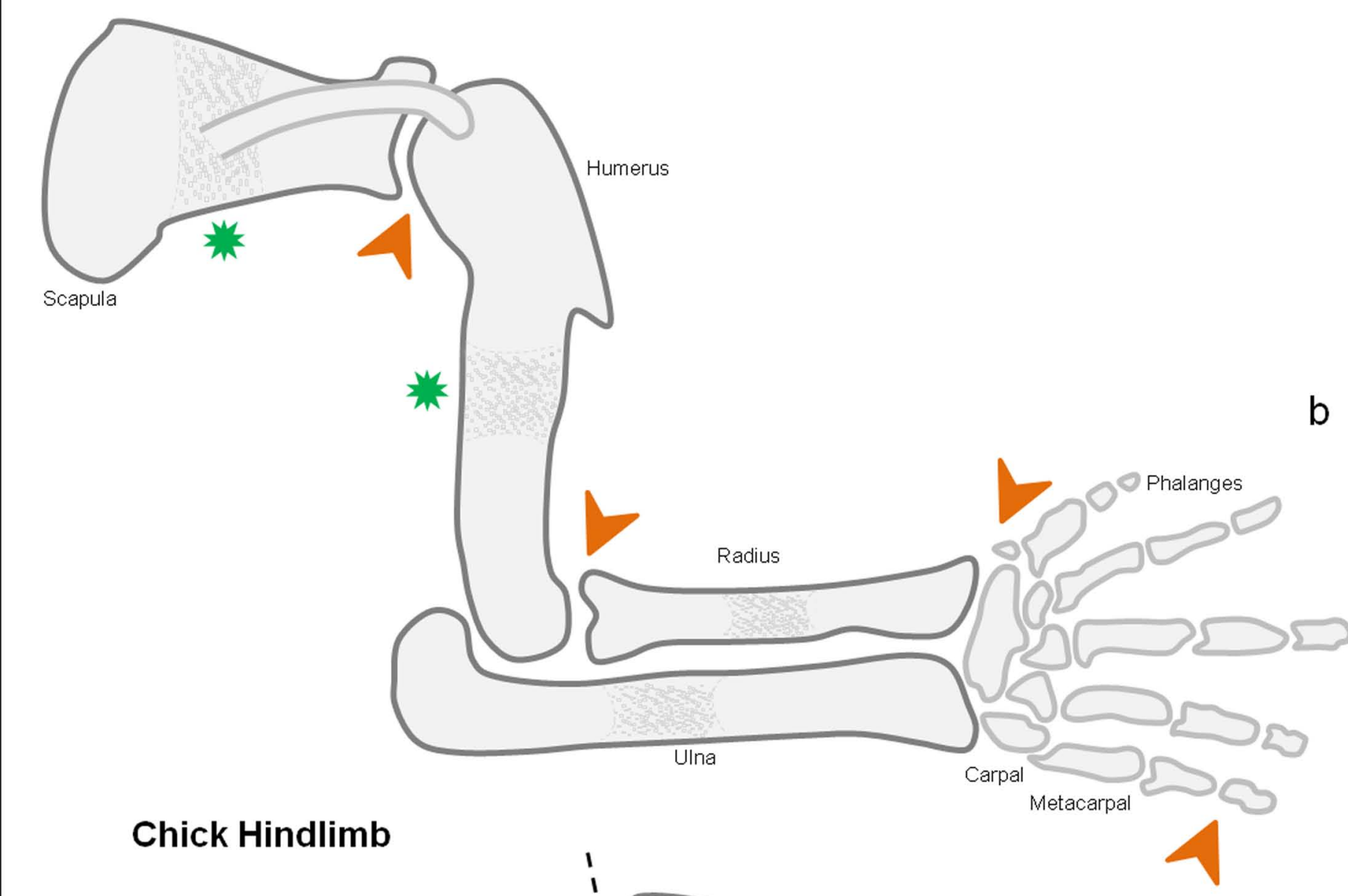

\section{Chick Hindlimb}

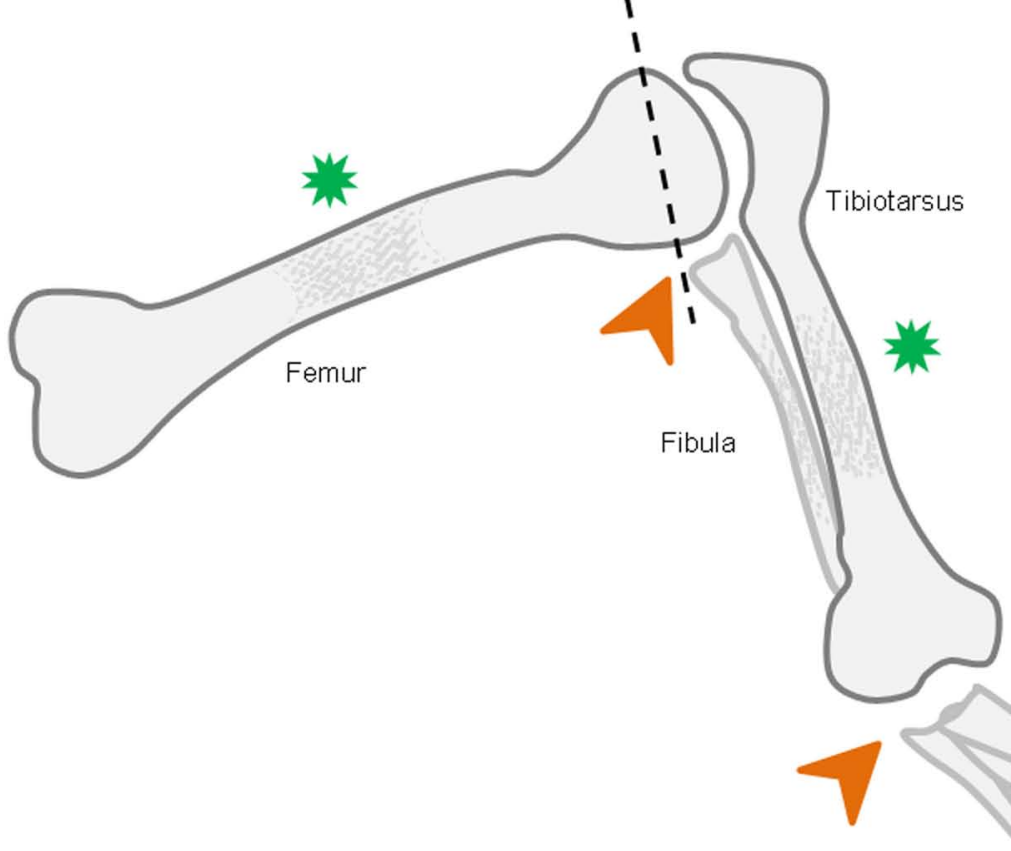

a
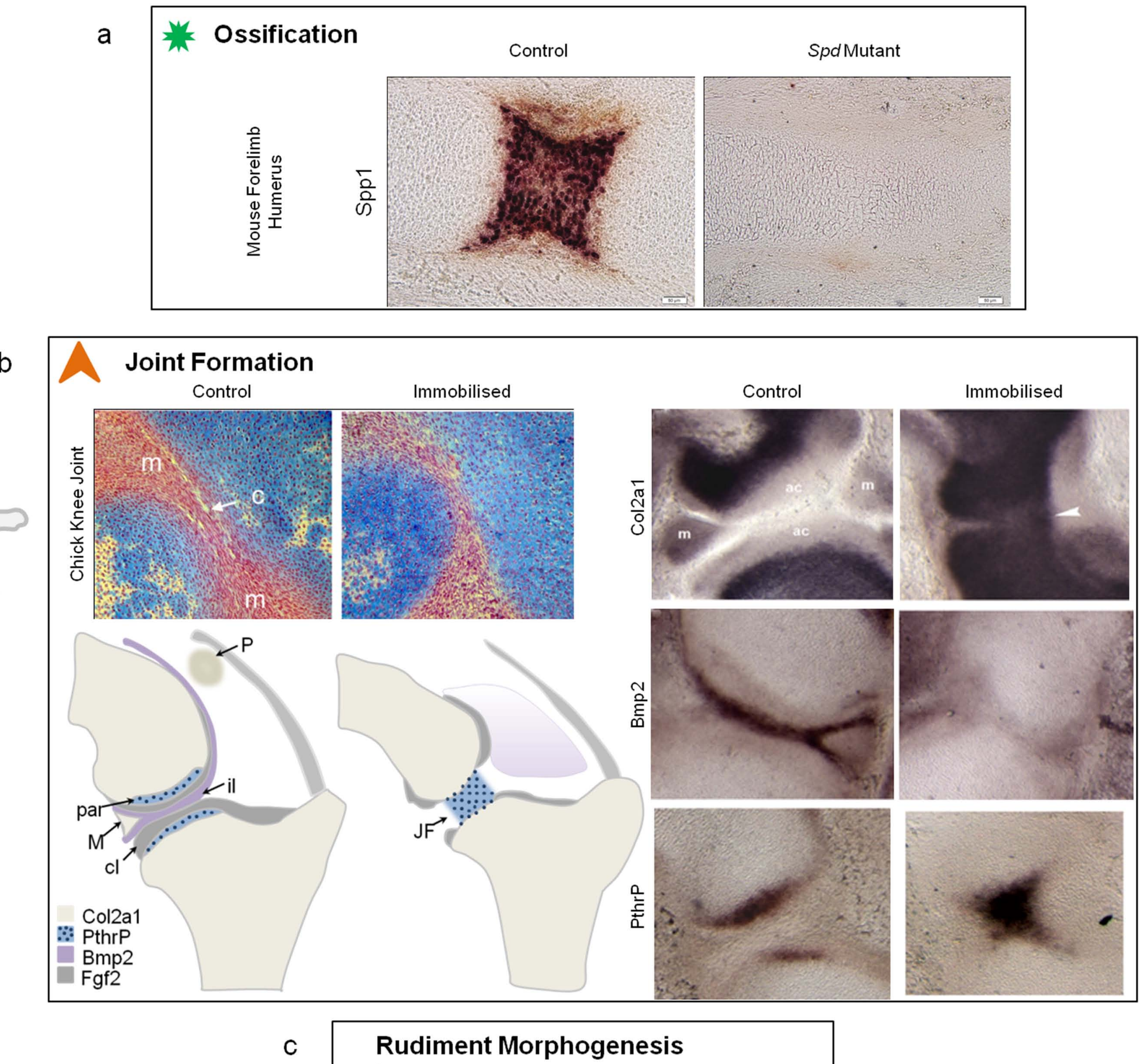

c

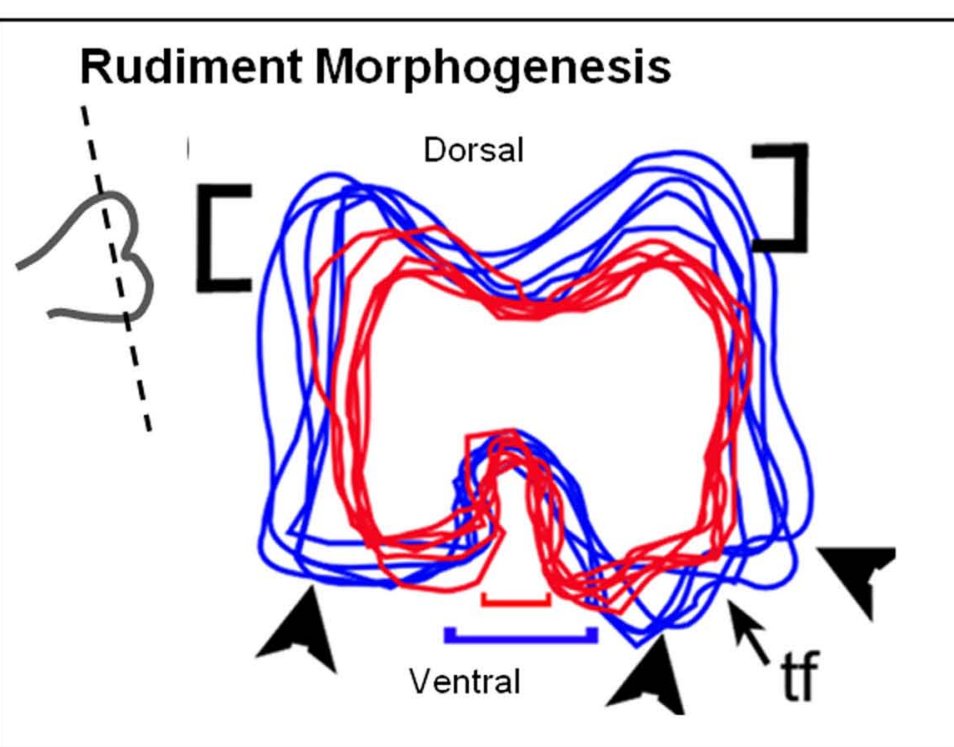

